\title{
A case of high grade pancreatic intraepithelial neoplasia diagnosed by endoscopic ultrasound-guided fine needle aspiration
}

We report a case of high grade pancreatic intraepithelial neoplasia diagnosed by endoscopic ultrasound-guided fine needle aspiration (EUS-FNA) ( $\triangleright$ Video 1 ). The patient was a 68-year-old man who came for a checkup. Main pancreatic duct (MPD) dilatation was observed on abdominal ultrasonography. His tumor marker levels were normal. Contrast-enhanced computed tomography showed a dilated caudal MPD and pancreatic parenchyma atrophy, consistent with the interruption of the MPD in the pancreatic body ( $\triangleright$ Fig. 1). Magnetic resonance cholangiopancreatography revealed caudal MPD dilatation and a small cystic lesion around the MPD stenosis. Endoscopic ultrasound (EUS) showed a faint 10-mm hypoechoic area around the pancreatic duct with indistinct borders, irregular contours, and heterogeneous internal echogenicity.

High grade pancreatic intraepithelial neoplasia or minimally invasive carcinoma was suspected. Endoscopic pancreatography revealed a $20-\mathrm{mm}$ stenosis in the MPD ( $\mathbf{F i g . 2 )}$; endoscopic nasopancreatic drainage and subsequent pancreatic juice cytology were performed [1]. All samples were classified as Class III. EUS-FNA was performed to evaluate the faint hypoechoic area in order to exclude malignancy ( $\mathbf{F i g} \cdot \mathbf{3}$ ). Four punctures were performed using EZ-shot3 plus (22G; Olympus Medical Systems Corp., Tokyo, Japan). The patient developed mild pancreatitis that improved with conservative treatment.

The pathological diagnosis was adenocarcinoma (> Fig.4). He underwent laparoscopic pancreatic tail resection with a preoperative diagnosis of T1aNOM0 stage I or TisNOMO stage 0. Microscopic examination revealed papillary growth of high grade pancreatic intraepithelial

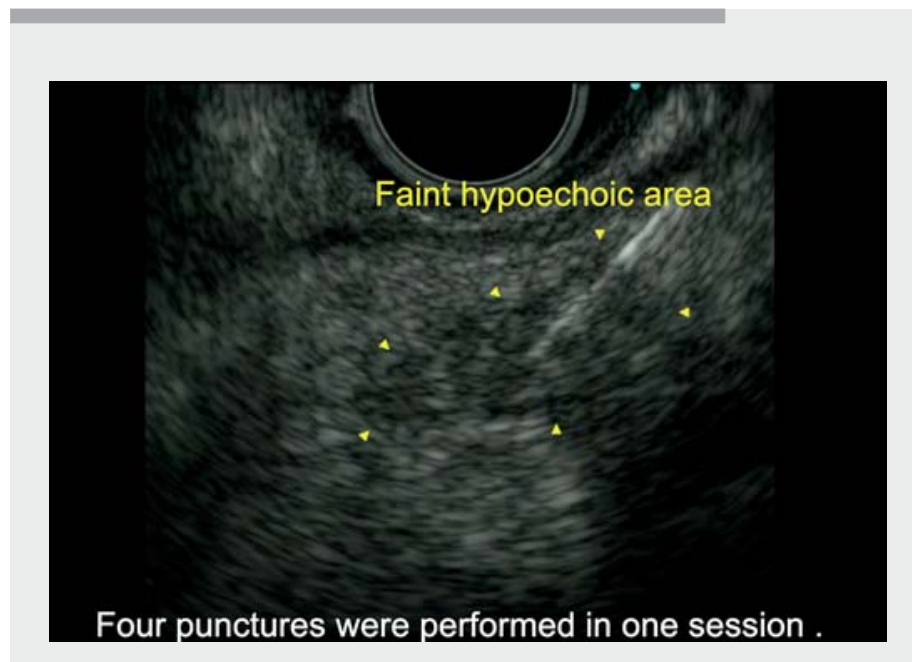

$\checkmark$ Video 1 We report a case of high grade pancreatic intraepithelial neoplasia diagnosed by endoscopic ultrasound-guided fine needle aspiration, which was difficult to diagnose using pancreatic juice cytology.
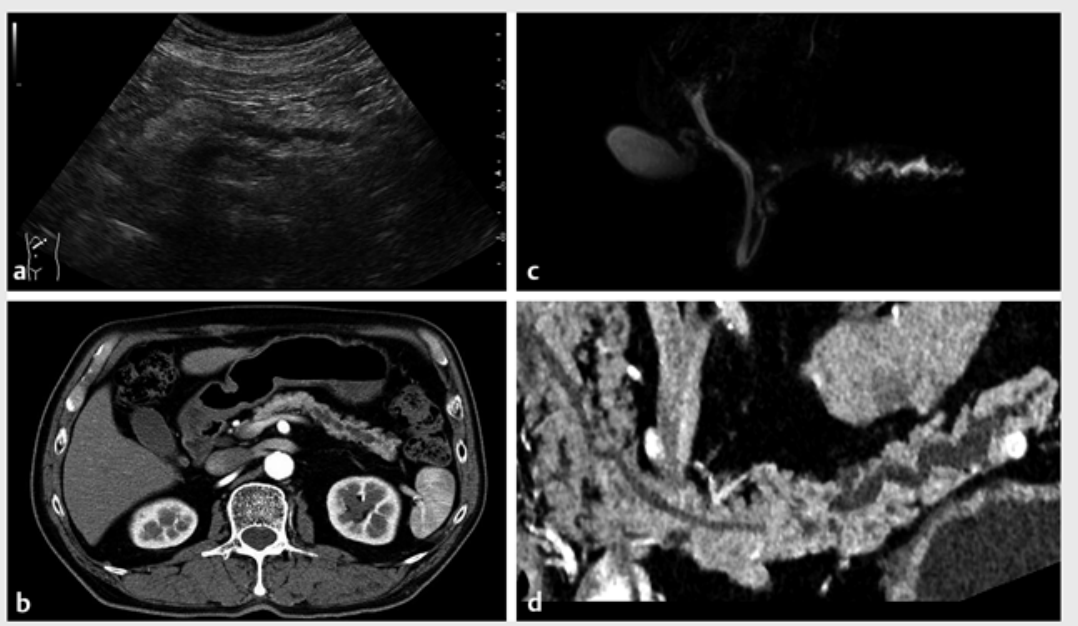

Fig. 1 a Abdominal ultrasonography showed stenosis of the main pancreatic duct in the pancreatic body and main pancreatic duct (MPD) dilatation. b Computed tomography (CT) demonstrated dilatation of the caudal MPD. Atrophy of the pancreatic parenchyma coincided with the interruption of the MPD in the pancreatic body. $c$ Magnetic resonance cholangiopancreatography showed dilatation of the caudal MPD and a small cystic lesion around the MPD stenosis in the body of the pancreas. These examinations did not reveal any obvious masses. $\mathbf{d}$ CT scan reconstructed with the main pancreatic duct in the axis. 

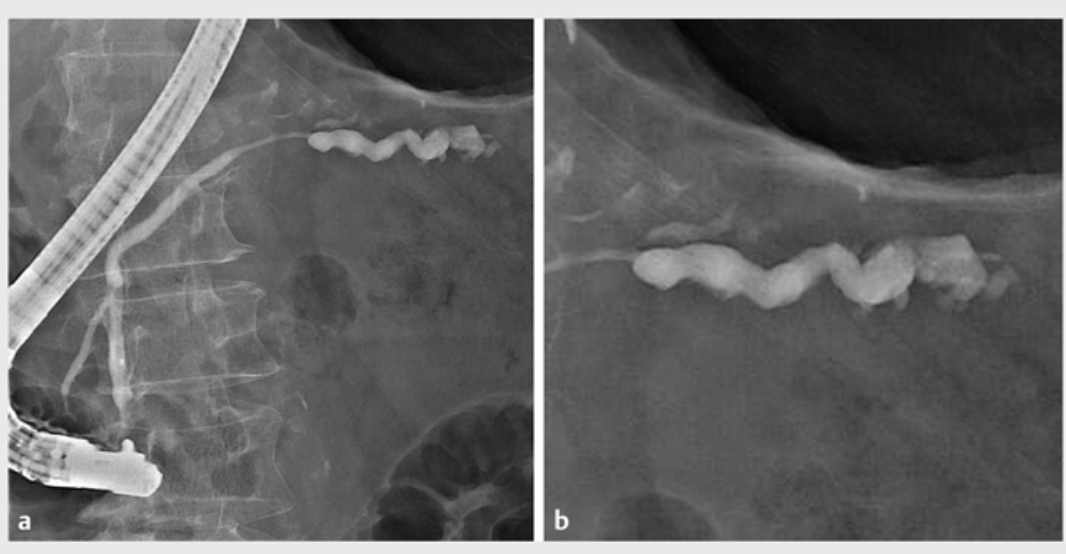

- Fig. 2 a Endoscopic pancreatography showed a 20-mm stenosis in the main pancreatic duct (MPD) of the pancreatic body. Dilatation of the caudal MPD was observed. $\mathbf{b}$ Dilation of the branch pancreatic duct was also observed caudal to the stenosis.
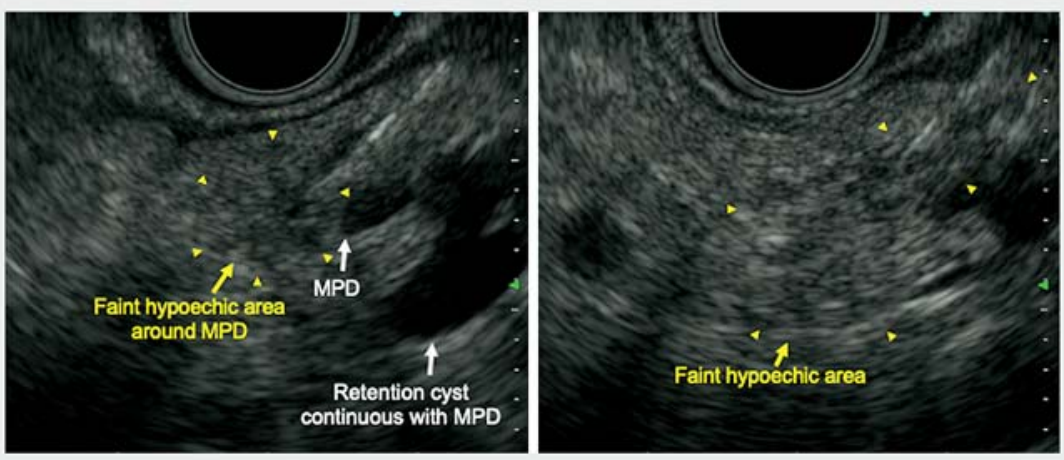

Fig. 3 Endoscopic ultrasound (EUS) showed wall thickening of the main pancreatic duct (MPD) in the body of the pancreas as well as a faint hypoechoic area with indistinct borders, irregular contours, and inhomogeneous internal echogenicity around the pancreatic duct. The faint hypoechoic area was continuous from the MPD to the periphery of the dilated branch pancreatic duct. EUS did not reveal any mass lesions.

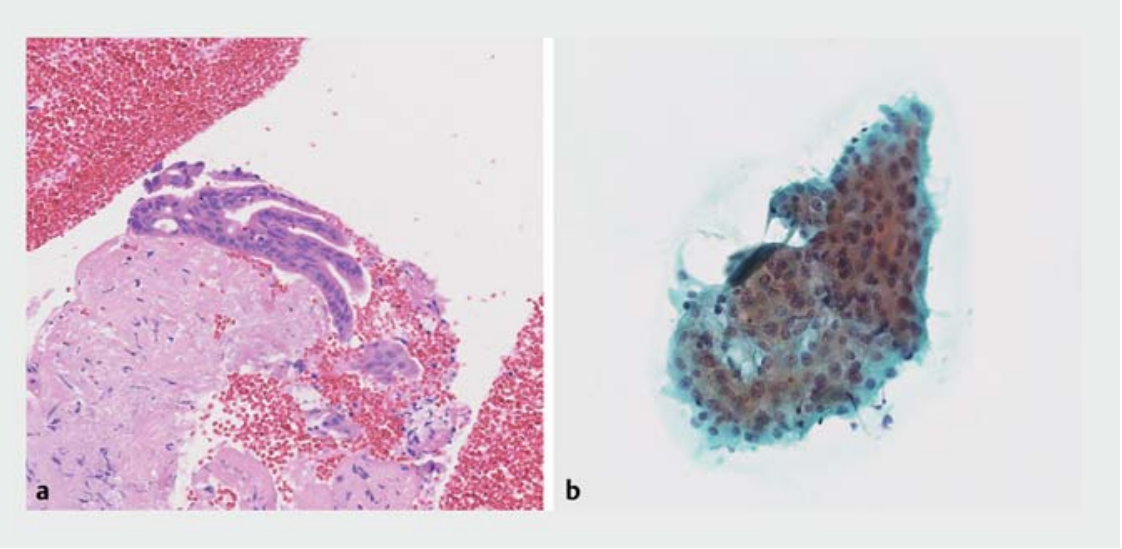

- Fig. 4 Tissue samples were collected by endoscopic ultrasound-guided fine needle aspiration. Both histology and cytology revealed adenocarcinoma. a Hematoxylin \& eosin staining, $10 \times$. b Papanicolaou's stain, $40 \times$. neoplasia in the branch of the pancreatic duct contiguous with the MPD ( $\bullet$ Fig. 5 ). There was no invasion of the pancreatic parenchyma. The final pathological diagnosis was TisNOMO stage 0 . We speculated that tumor cells could be confirmed by EUS-FNA because of the widespread presence of the high grade pancreatic intraepithelial neoplasia in the branched pancreatic duct and the weak cell adhesion of the cancer. There has been only one previous report of high grade pancreatic intraepithelial neoplasia diagnosed by EUS-FNA [2]. We encountered a high grade pancreatic intraepithelial neoplasia case diagnosed by EUS-FNA as a rescue for negative pancreatic juice cytology.

Endoscopy_UCTN_Code_TTT_1AS_2AF

Funding

The National Cancer Center Research and Development Fund

31-A-13

Japanese Foundation for Research and Promotion of Endoscopy

http://dx.doi.org/10.13039/100018254

Competing interests

The authors declare that they have no conflict of interest.

The authors

Hidetoshi Kitamura', Susumu Hijioka', Yoshikuni Nagashio ${ }^{1}$, Daisuke Ban ${ }^{2}$, Minoru Esaki $^{2}$, Takuji Okusaka ${ }^{1}$, Yutaka Saito ${ }^{3}$

1 Department of Hepatobiliary and Pancreatic Oncology, National Cancer Center Hospital, Tokyo, Japan

2 Department of Hepatobiliary and Pancreatic Surgery, National Cancer Center Hospital, Tokyo, Japan

3 Endoscopy Division, National Cancer Center Hospital, Tokyo, Japan

\section{Corresponding author}

\section{Susumu Hijioka, MD}

Department of Hepatobiliary and Pancreatic Oncology, National Cancer Center Hospital, 5-1-1 Tsukiji, Chuo-ku, Tokyo 104-0045, Japan

Fax: +81-3-3542-3815

shijioka@ncc.go.jp 


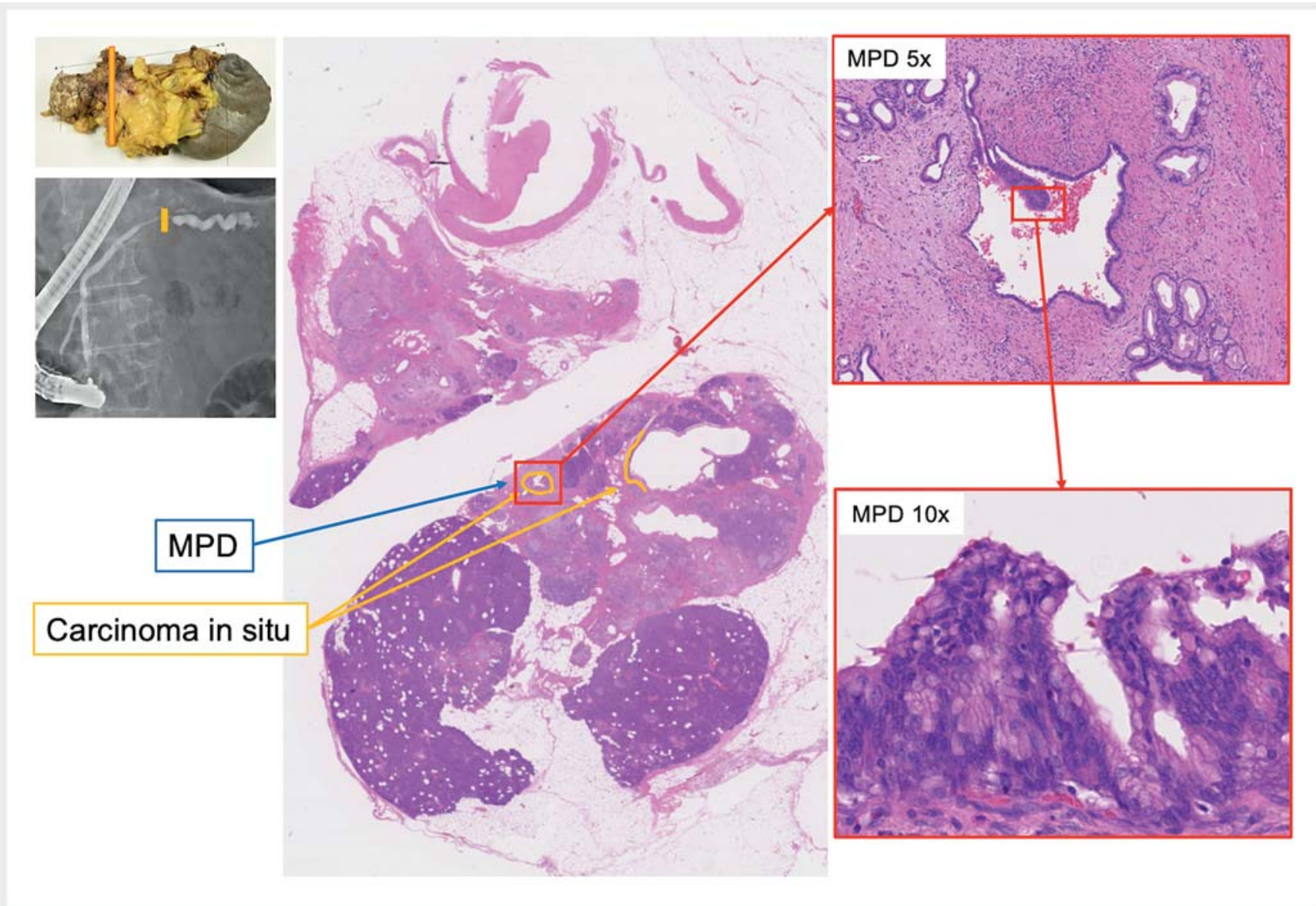

Fig. 5 Tumor cells with a high nuclear-to-cytoplasmic ratio with papillary growths in the pancreatic duct. There was no invasion into the pancreatic parenchyma, and the tumor was confined to the epithelium of the pancreatic duct, indicating high grade pancreatic intraepithelial neoplasia. It was extensively present from the main pancreatic duct to the branch ducts.

\section{References}

[1] liboshi T, Hanada K, Fukuda T et al. Value of cytodiagnosis using endoscopic nasopancreatic drainage for early diagnosis of pancreatic cancer: establishing a new method for the early detection of pancreatic carcinoma in situ. Pancreas 2012; 41: 523-529

[2] Sakamoto H, Kitano M, Dote K et al. In situ carcinoma of pancreas diagnosed by EUSFNA. Endoscopy 2008; 40: E15-E16

\section{Bibliography}

Endoscopy 2022; 54: E628-E630

DOI $10.1055 / \mathrm{a}-1730-3973$

ISSN 0013-726X

published online 4.2 .2022

(C) 2022. Thieme. All rights reserved.

Georg Thieme Verlag KG, Rüdigerstraße 14, 70469 Stuttgart, Germany

ENDOSCOPY E-VIDEOS
https://eref.thieme.de/e-videos
Endoscopy E-Videos is an
and new techniques in gastroenterological
endoscopy. All papers include a high
quality video and all contributions are
freely accessible online. Processing charges
apply (currently EUR 375), discounts and
wavers acc. to HINARI are available.
This section has its own submission
website at
https://mc.manuscriptcentral.com/e-videos

